\title{
Off-axis primary-dose measurements using a mini-phantom.
}

\author{
Stefan A. Johnsson \\ Department of Radiation Physics \\ Lund University Hospital \\ S-221 85 Lund \\ Sweden
}

[Submitted to Medical Physics (October 1996)]

Abstract 
The characterization of the incident photon beam is usually divided into its dependence on collimator setting (head-scatter factor) and off-axis position (primary off-axis ratio). These parameters are normally measured "in air" with a build-up cap, thick enough to generate full dose build-up at the depth of dose maximum. In order to prevent any influence from contaminating electrons, it has been recommended that head-scatter measurements are carried out using a mini-phantom rather than a conventional buildup cap. Due to the volume of the mini-phantom, the effects from attenuation and scatter are not negligible. In relative head-scatter measurements these effects cancel and the head-scatter is thus a good representation of the variation of the incident photon beam with collimator setting. However, in off-axis measurements, attenuation and scatter conditions vary due to beam softening and do not cancel in the calculation of the primary off-axis ratio. The purpose of the present work was to estimate the effects from attenuation and phantom-scatter in order to determine their influence on primary off-axis ratio measurements. We have characterized the off-axis beam softening effect by means of narrow-beam transmission measurements to obtain the effective attenuation coefficient as a function of off-axis position. We then used a semianalytical expression for the phantom-scatter calculation that depends solely on this attenuation coefficient. The derived formalism for relative "in air" measurements using a mini-phantom is clear and consistent, which enables the user to separately calculate the effects from scatter and attenuation. For the investigated beam qualities, 6- and 18MV, our results indicate that the effects from attenuation and scatter in the miniphantom nearly cancel (the combined effect is less than 1\%) within $12.5 \mathrm{~cm}$ from the central beam axis. Thus, no correction is needed when the primary off-axis ratio is measured with a mini-phantom.

Keywords: off-axis ratio, primary dose, mini-phantom, phantom-scatter, beam softening

\section{Introduction}


When calculating the photon dose in teletherapy it is sometimes attractive to separate the total dose, $\mathrm{D}$, into primary, $\mathrm{P}$, and scatter dose, $\mathrm{S}$, according to: ${ }^{1,2}$ $D=P+S=P \cdot(1+S P R)=P \cdot S F$

where SPR is the scatter-to-primary ratio and SF is the scatter factor. The primary dose is determined by the incident photon beam and can be further divided into its dependence on depth, d, collimator setting, c, and off-axis distance, r:

$P=A(d) \cdot H(c) \cdot O A R(r)$

where $\mathrm{A}, \mathrm{H}$ and OAR are abbreviations for attenuation, head-scatter factor and offaxis ratio, respectively. The off-axis ratio is defined as the ratio between the primary dose off and on the central axis, sometimes also named off-center ratio or primary offcenter ratio. $^{3-7}$ This ratio is required for calculations in asymmetric fields where the calculation point is not on the central axis. Eq. (2) presumes that $\mathrm{H}$ and OAR are mutually independent and that electron equilibrium is present.

Both the head-scatter factor and the off-axis ratio should be measured "in air" under conditions where there is no scatter contribution from the measurement device. In practice, a small inevitable scatter contribution can be tolerated as long as it cancels out in relative measurements. Such measurements are normally performed with a buildup cap, thick enough to generate full dose build-up at the depth of dose maximum. However, there are some drawbacks with this technique; ${ }^{8-11}$

a) Contaminating electrons from the treatment head and the air may contribute to the measured dose.

b) The depth of the dose maximum may vary with field size and beam modifying devices.

c) To be applicable in small field sizes, a high-Z build-up cap has to be used. This is undesirable because of different interaction properties between high- $Z$ materials and water.

To avoid these problems it has been recommended ${ }^{8,12}$ that "in-air" measurements are carried out at depths exceeding the depth of maximum dose, using a mini-phantom rather than a conventional build-up cap. Comparisons between these two methods for head-scatter measurements can be found in the literature. ${ }^{8,14}$

In this paper we investigate the feasibility of using a mini-phantom also for off-axis measurements. Possible effects of off-axis beam softening ${ }^{13,18}$, leading to altered 
attenuation and scatter conditions in the mini-phantom, are examined using models based on the effective attenuation coefficient, ${ }^{15-17}$ which is measured in a narrow beam geometry. $^{19}$

\section{Theory}

Relative dose measurements using the mini-phantom may according to Eqs. (1)-(2) be expressed as:

$$
\frac{D(d, c, r)}{D\left(d_{r e f}, c_{r e f}, r_{r e f}\right)}=\frac{A(d) \cdot H(c) \cdot O A R(r) \cdot S F(d, s)}{A\left(d_{r e f}\right) \cdot H\left(c_{r e f}\right) \cdot O A R\left(r_{r e f}\right) \cdot S F\left(d_{r e f}, s_{r e f}\right)}
$$

where index "ref" denotes reference conditions. Due to the volume of the miniphantom, the effects from attenuation and scatter are not negligible. The scatter factor applies to the irradiated volume defined by the shape and the size of the mini-phantom, see Fig. (1). The detector is positioned at depth $d=d_{\text {ref }}=5 \mathrm{~cm}$, where the size is $s=s_{\text {ref }}=4$ $\mathrm{cm}$. In Eq. (3), both A and SF are also functions of beam quality as it varies with offaxis distance $r$, and we have:

$$
\frac{D(c, r)}{D_{r e f}}=\frac{A(r)}{A(0)} \cdot \frac{S F(r)}{S F(0)} \cdot H(c) \cdot O A R(r)
$$

Here $\mathrm{H}\left(\mathrm{c}_{\mathrm{ref}}=10\right)$ and $\mathrm{OAR}\left(\mathrm{r}_{\mathrm{ref}}=0\right)$ are both set to unity. For head-scatter measurements $\mathrm{c}$ is varied but $\mathrm{r}=0$ and Eq. (4) reduces to:

$$
\frac{D(c)}{D_{r e f}}=H(c)
$$

Thus, head-scatter can be measured with the mini-phantom since the collimator setting does not affect the energy spectrum. For off-axis measurements, on the other hand, $r$ is varied while $\mathrm{c}=\mathrm{c}_{\mathrm{ref}}=40 \mathrm{~cm}$, and we have:

$$
\frac{D(r)}{D_{r e f}}=\frac{A(r)}{A(0)} \cdot \frac{S F(r)}{S F(0)} \cdot O A R(r)=k(r) \cdot O A R(r)
$$

Thus, if $\mathrm{k}(\mathrm{r})$ is known, OAR can be determined by relative dose measurements using a mini-phantom.

The relative effect from attenuation is simply: 


$$
\frac{A(r, d)}{A(0, d)}=\frac{e^{-\mu(r) \cdot d}}{e^{-\mu(0) \cdot d}}
$$

For the calculation of the scatter factor we use a semi-empirical expression derived by Bjärngard et.al., which has shown to be in good agreement with measured scatter factors, especially for small field sizes and at shallow depths: ${ }^{15-17}$

$$
S F(d, s)=1+a \cdot \frac{s \cdot d}{w \cdot s+d}
$$

where $s$ is the field size at depth d. Note that the volume of our "beam-divergent" mini-phantom is the same as the irradiated volume of a large phantom at a depth of 5 $\mathrm{cm}$ and with a field size of $4 * 4 \mathrm{~cm}^{2}$. Therefore, it is possible to use Eq. (8) for calculation of the scatter factor in the mini-phantom.

The parameters a and $\mathrm{w}$ depend on $\mu$, according to: ${ }^{16}$

$$
\mathbf{a}=\mathbf{1 . 1 7} \boldsymbol{\mu - 0 . 0 0 9 3}
$$

$$
\mathrm{w}=1.44-14.8 \mu
$$

Thus, given the parameters $s$ and $d$, we only need $\mu(r)$ to calculate both attenuation and scatter in the mini-phantom at off-axis positions. The combined effect is defined by Eq. (6) and denoted by k(r).

Consequently, OAR can be derived by dividing the dose ratio measured with a miniphantom with $\mathrm{k}$, and the result would then be the primary dose off-axis ratio.

\section{Materials and Methods}

All measurements were performed with 6-MV and 18-MV X-ray beams from a Philips SL25 linear accelerator at Lund University Hospital.

\section{A. Off-axis measurements with the mini-phantom}

The mini-phantom is divergent with the fan-lines of the beam and is made of polystyrene (white), with a relative electron density compared to water of 1.04 , see Fig. (1). The mini-phantom is placed on the treatment couch in a Styrofoam holder to minimize any influence from backscatter. The mini-phantom is combined with both an ionization chamber (Scanditronix RK8305) and an energy compensated diode (Scanditronix Photon diode type 2). For comparison, off-axis measurements were also made with an ionization chamber (NE2571) in combination with a build-up cap made 
of perspex. The caps were chosen thick enough, $1.5 \mathrm{~cm}$ and $3.2 \mathrm{~cm}$, to generate full build-up at 6-MV and 18-MV, respectively. Note that full build-up is with regard to electrons generated in the medium, the build-up cap is not necessarily thick enough to fully attenuate contaminating electrons. The off-axis dose ratio, $\mathrm{D}(\mathrm{r}) / \mathrm{D}_{\text {ref }}$, was measured in a $40 * 40 \mathrm{~cm}^{2}$ field with a source-detector distance (SDD) of $100 \mathrm{~cm}$ at the central axis. The detector was moved perpendicular to the central beam axis, in the direction perpendicular to the gun-target direction (A-B direction), a distance $r$.

One conceivable disadvantage by moving the mini-phantom in a perpendicular direction to the central axis is that the photons will have an oblique incidence. Testing of these possible misalignment effects was performed by comparing results from these measurements with measurements where the mini-phantom, with the diode, was tilted towards the target as described in Fig. (2).

\section{B. $\mu$-measurements and calculation of $A$ and SF in the mini-phantom}

At off-axis positions there is beam softening of the incident (primary) beam due to the shape of the flattening filter and to inherent properties in the production of brehmsstrahlung. It is possible to describe this energy degradation with the effective attenuation coefficient, $\mu(\mathrm{r})$, which is an effective mean value for the actual energy spectrum, as a function of off-axis position. Measurements of the transmission factors, A, through water $(x=26.8 \mathrm{~cm})$ in a narrow beam configuration givs the parameter $\mu(\mathrm{r})$, solving Eq. (11), for different off-axis positions. ${ }^{19}$

$$
A(x+\delta)=A(\delta) \cdot e^{-\mu(r) x}
$$

Here $\delta$ is the depth of the detector $(5 \mathrm{~cm})$ in a polystyrene phantom. We have ignored beam hardening in Eq. (11) since the effect is small. To accomplish a "non-scatter condition" the gantry was set to $90^{\circ}$ and the patient support assembly was aligned with the beam direction. A plastic water tank was placed on the patient support assembly at the isocenter, and the phantom, with a diode, at the other end about $2.5 \mathrm{~m}$ away from the target. This, together with a small field size, minimized the effects of scatter to a degree where it could be assumed to be negligible. The field size at isocenter, $s=c=2$ $\mathrm{cm}$, fully covers the phantom at $2.5 \mathrm{~m}$, thus all the criteria for a narrow beam geometry are fulfilled. The water thickness include the plastic walls of the tank, converted to water equivalent depth. 
Measurement at off-axis positions in the A-B direction were made according to Fig. (3). With this method the limitation in maximum off-axis distance $(12.5 \mathrm{~cm})$ is due to the limited collimator off-set when using small field sizes. This problem can be avoided with an external collimator in combination with a large field size.

The calculations of attenuation, scatter and the combined effect, $\mathrm{k}$, in the miniphantom were then accomplished by using Eqs. (6)-(10).

\section{Results}

\section{A. Off-axis measurements with the mini-phantom}

An increase in $\mathrm{D} / \mathrm{D}_{\text {ref }}$ with off-axis distance was measured, reaching $5 \%$ at $12.5 \mathrm{~cm}$. There was no significant difference between the diode and the ionization chamber nor between aligned and misaligned mini-phantom (less than $0.4 \%$ ). Neither was there any difference (less than $0.5 \%$ ) between the mini-phantom and the build-up cap.

\section{B. $\mu$-measurements and calculations of $\mathrm{A}$ and $\mathrm{SF}$ in the mini-phantom}

The measured $\mu$-values are plotted in Figs. (4) and (5) for 6-MV and 18-MV, respectively, with a second order polynomial fitted to the values. These equations are used for the calculation of scatter and attenuation in the mini-phantom, which are plotted in Figs. (6) and (7), together with the combined effect k, as a function of offaxis distance. The results at maximum $\mathrm{r}=12.5 \mathrm{~cm}$ are summarized in Table I.

\section{Discussion}

There are several potential advantages of using a mini-phantom rather than a buildup cap for OAR measurements. First, it reduces the influence of contaminating electrons. In our particular case, however, we see no significant discrepancy between the mini-phantom and the build-up cap; less than $0.5 \%$. Second, the consistency with the proposed method of choice for head scatter measurements is desirable. There is no reason for using two different techniques. Finally, and maybe most important, through Eq. (4) we have a congruous semi-empirical formalism for relative dose measurements using the mini-phantom, which does not exist when using a build-up cap.

We have found the mini-phantom reliable and easy to use. The maximal deviation due to misalignment of the mini-phantom relative to the beam was less than $0.4 \%$, and 
there was no significant difference between the two detector systems (diode and ionization chamber).

However, the shift in attenuation and scatter could possibly affect the result. These effects were calculated with Eqs. (7)-(11) where the only parameter needed was the effective attenuation coefficient, $\mu(r)$, at different off-axis positions, determined in narrow beam measurements. Assuming radial beam symmetry, the $\mu(\mathrm{r})$ measured in the A-B direction can be used for the entire field. A more accurate description of the incident photon beam would be to make a map of $\mu(\mathrm{r})$, including a matrix of points covering the field. Our measured $\mu$ extends only to a distance of $12.5 \mathrm{~cm}$ which of course is a limitation. This may be avoided by using external blocks in combination with a large field size.

The results of the calculation of attenuation and scatter in the mini-phantom showed that the attenuation decreases and the scatter increases with off-axis positions. The combined effect, $\mathrm{k}$, is close to unity for 6-MV and less than $1 \%$ for $18-\mathrm{MV}$ within $12.5 \mathrm{~cm}$ from the central axis. Thus, there seems to be no need to correct for the combined effect inside a $12.5 \mathrm{~cm}$ radius.

The final statement we would like to make is a caution. There can sometimes be a temptation in making a correction for the shift in attenuation when going off-axis, since this is rather easy. But doing this without any consideration to the shift in scatter properties can do more damage than good, due to the fact that the two effects are in opposite directions.

\section{Conclusions}

When using a mini-phantom for primary dose measurements the results are influenced by beam softening, which implies changes in both attenuation and scatter properties. We have demonstrated that these effects can be quantified using semiempirical formulas. The attenuation effects amounts to nearly $2 \%$, but since the scatter effect goes in the opposite direction our conclusion is that no correction is needed within a $12.5 \mathrm{~cm}$ radius of the central axis of the beam.

\section{Acknowledgements}


The authors would like to acknowledge Dr. P. Nilsson for his useful criticism on this manuscript and Kurt Larsson for the making of the mini-phantom.

Table I. The relative effects from attenuation, scatter and the combined effect, $\mathrm{k}$, at $12.5 \mathrm{~cm}$.

\begin{tabular}{c|cc}
\hline \hline & 6-MV & 18-MV \\
\hline $\mathbf{A}(\mathbf{1 2 . 5}) / \mathrm{A}(\mathbf{0})$ & 0.985 & 0.981 \\
$\mathbf{S F}(\mathbf{1 2 . 5}) / \mathrm{SF}(\mathbf{0})$ & 1.010 & 1.011 \\
$\mathbf{k}(\mathbf{1 2 . 5})$ & 0.995 & 0.992 \\
\hline \hline
\end{tabular}

\section{References}

${ }^{1}$ R. Mohan and C. Chui, "Validity of the concept of separating primary and scatter dose," Med. Phys. 12, 726-730 (1985).

${ }^{2}$ B. E. Bjärngard, H. Rashid and C. H. Obcemea, "Separation of primary and scatter components of measured photon beam data," Phys. Med. Biol. 34, 1939-1945 (1989). ${ }^{3}$ C. Chui and R. Mohan, "Off-center ratios for three-dimensional dose calculations," Med. Phys. 13, 409-412 (1986).

${ }^{4}$ J. P. Gibbons and F. M. Kahn, "Calculation of dose in asymmetric photon fields," Med. Phys. 22, 1451-1457 (1995).

${ }^{5}$ D. D. Loshek and K. A. Keller, "Beam profile generator for asymmetric fields," Med. Phys. 15, 604-610 (1988).

${ }^{6}$ F. M. Kahn, B. J. Gerbi, and F. C. Deibel, "Dosimetry of asymmetric x-ray collimators," Med. Phys. 13, 936-941 (1986).

${ }^{7}$ D. D. Loshek and T. T. Parker, "Dose calculation in static or dynamic off-axis fields," Med. Phys. 21, 401-410 (1994). 
${ }^{8}$ J. J. Van Gasteren, S. Heukelom, R. van der Laarse, J. L. M. Venselar, and C. F. Westerman, "The determination of phantom and scatter components of the output of megavoltage photon beams: Measurements of collimator scatter part with a beam coaxial narrow cylindrical phantom," Radiother. Oncol. 20, 250-257 (1991).

${ }^{9}$ B. E. Bjärngard, P. Vadash, and T. Zhu, "Doses near the surface in high-energy x-ray beams," Med. Phys. 22, 465-468 (1995).

${ }^{10}$ B. R. Thomadsen, S. S. Kubsad, B. R. Paliwal, S. Shahabi, and T. R. Mackie, “On the cause of the variation in tissue-maximum ratio values with source-to-detector distance," Med. Phys. 20, 723-727 (1993).

${ }^{11}$ G. Krithivas and S. N. Rao, "A study of characteristics of radiation contaminants within a clinically useful photon beam,” Med. Phys. 12, 764-768 (1985).

${ }^{12}$ B. J. Mijnheer, A. H. Aalbers, A. G. Visser, and F. W. Wittkamper, "Consistency and simplicity in the determination of absorbed dose to water in high-energy photon beams: a new code of practice," Radiother. Oncol. 7, 371-384 (1986).

${ }^{13}$ H. Nystöm and M. Karlsson, "Photon beam quality specification by narrow-beam transmission measurements," Phys. Med. Biol. 39, 1231-1245 (1994).

${ }^{14}$ D. M. D. Frye, B. R. Paliwal, B. R. Thomadsen, and P. Jursinic, "Intercomparison of normalized head-scatter factor measurement techniques," Med. Phys. 22, 249-253 (1995).

${ }^{15}$ B. E. Bjärngard, "Scatter factors for a 25-MV x-ray beam,” Med. Phys. 20, 357-361 (1993).

${ }^{16}$ B. E. Bjärngard and P. Vadash, "Analysis of central-axis doses for high-energy $\mathrm{x}$ rays," Med. Phys. 22, 1191-1195 (1995).

${ }^{17}$ B. E. Bjärngard and P. L. Petti, "Description of the scatter component in photonbeam data," Phys. Med. Biol. 33, 21-32 (1988).

${ }^{18}$ S. Zefkili, C. Kappas, and J. Rosenwald, "On-axis and off-axis primary dose component in high energy photon beams," Med. Phys. 21, 799-808 (1994).

${ }^{19}$ B. E. Bjärngard and H. Shackford, "Attenuation in high-energy x-ray beams," Med. Phys. 21, 1069-1073 (1994).

\section{Figure legends}


Fig. 1. The mini-phantom made of polystyrene with the detector at a depth of $5 \mathrm{~cm}$ where the crossection is $4 * 4 \mathrm{~cm}^{2}$. The shape of the phantom follows the lines of divergence from the source, assuming that the detctor is at isocenter.

Fig. 2. Setup for misalignment check.

Fig. 3. Geometry for alignment of the beam at off-axis positions. The detector is shown at isocenter for simplicity, but measurements at another distance will result in the same collimator, gantry and table movements, $\alpha=\tan ^{-1}\left(\frac{r}{100}\right)$ and $h=r \cdot \cos (\alpha)$.

Fig. 4. Measured $\mu$-values and a second order polynomial fitted to the measured values for 6-MV. The error bars result from a $0.3 \%$ short time drift.

Fig. 5. Measured $\mu$-values and a second order polynomial fitted to the measured values for 18-MV. The error bars result from a $0.3 \%$ short time drift.

Fig. 6. Calculated values of $\mathrm{SF}(\mathrm{r}) / \mathrm{SF}(0), \mathrm{A}(\mathrm{r}) / \mathrm{A}(0)$ and $\mathrm{k}(\mathrm{r})$ for 6-MV.

Fig. 7. Calculated values of $\mathrm{SF}(\mathrm{r}) / \mathrm{SF}(0), \mathrm{A}(\mathrm{r}) / \mathrm{A}(0)$ and $\mathrm{k}(\mathrm{r})$ for $18-\mathrm{MV}$. 

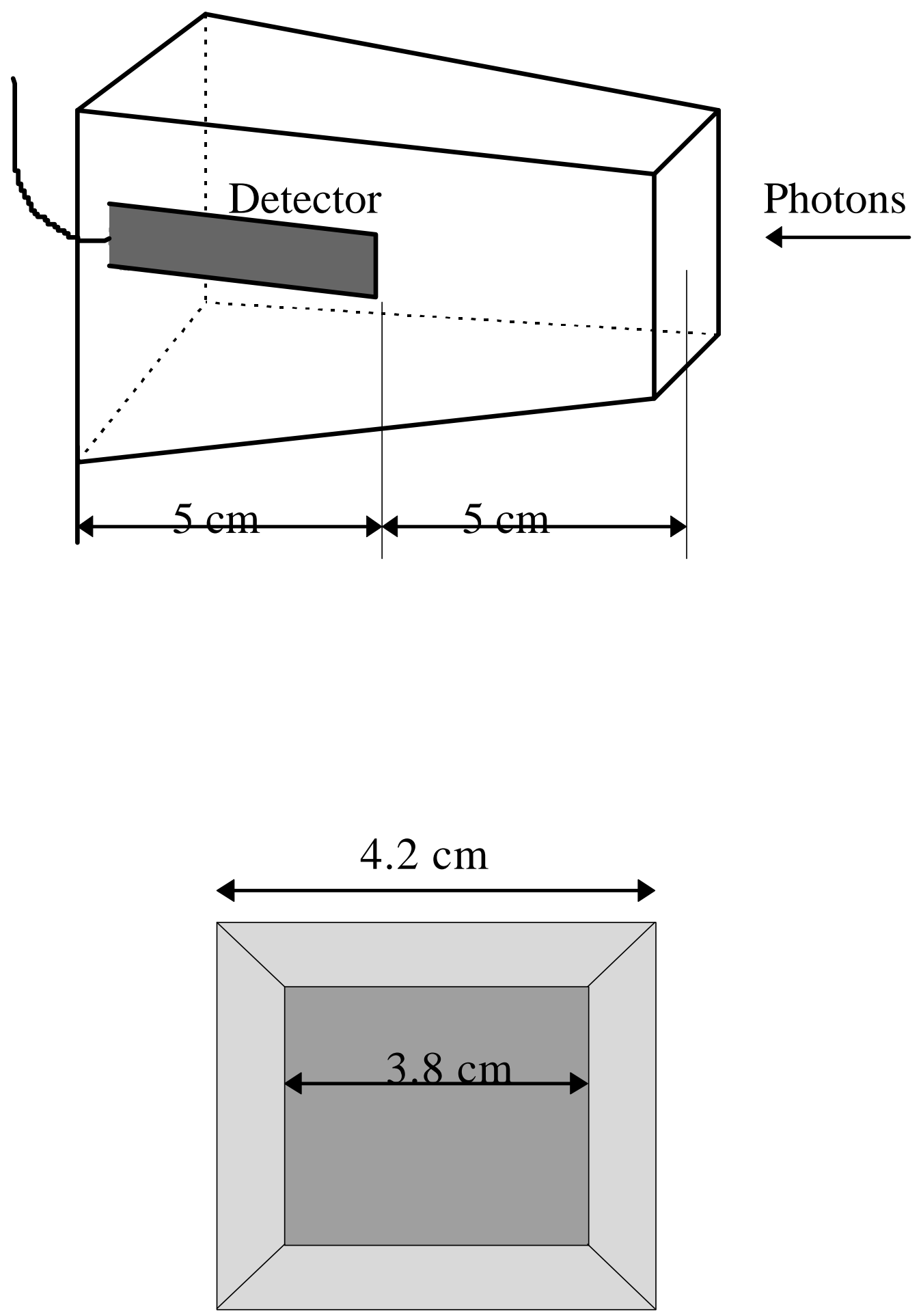


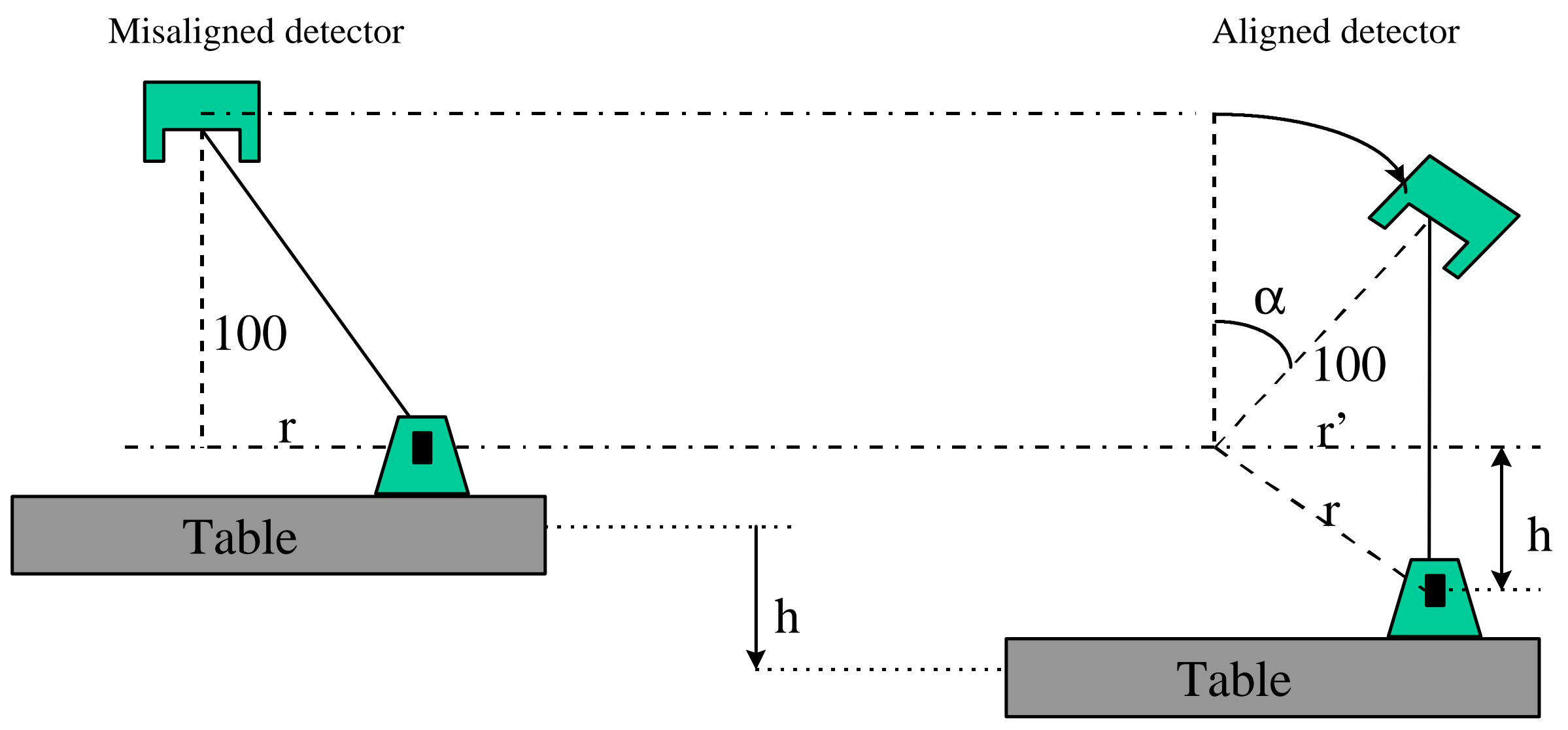


Figure 3.

1. On the central axis.

Collimators

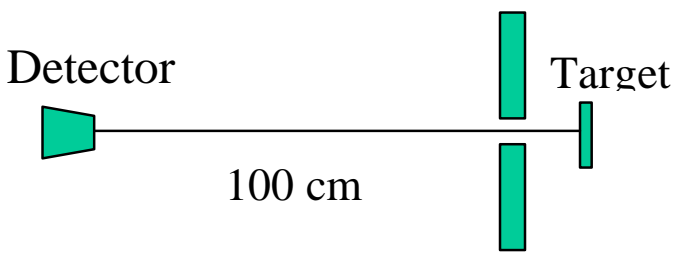

3. Gantrv rotated $\alpha$.

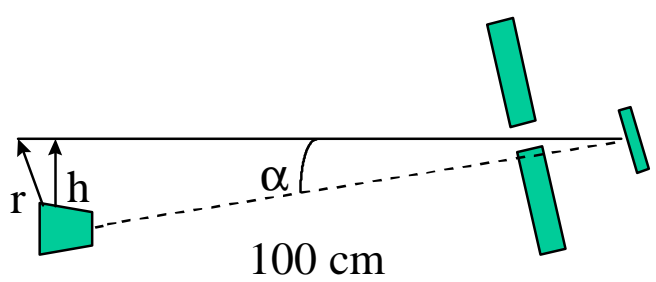

2. Collimator off-set.

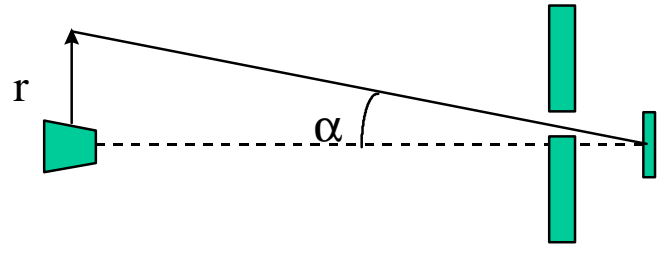

4. Table moved h.

Off the central axis.

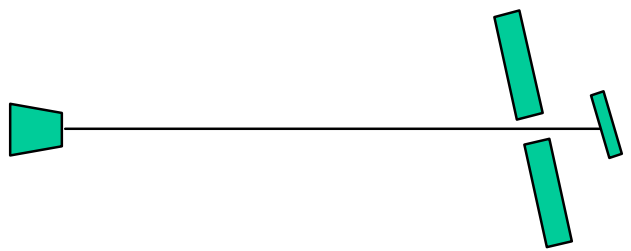


Figure 4.

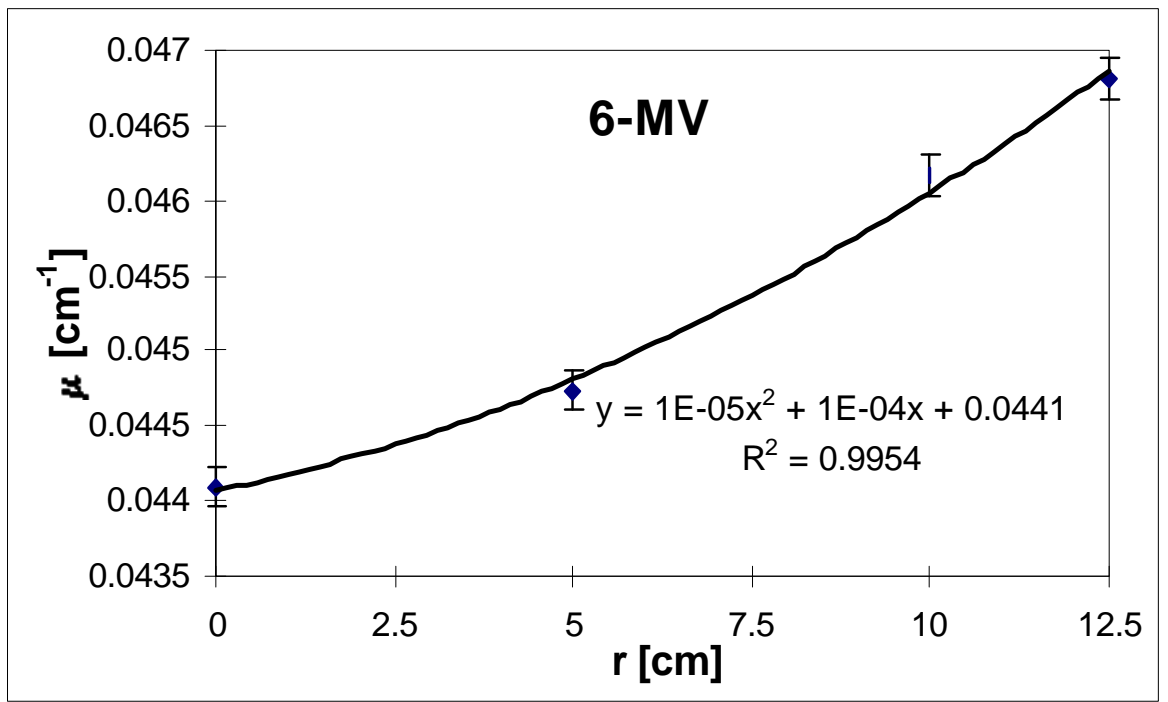

Figure 5.

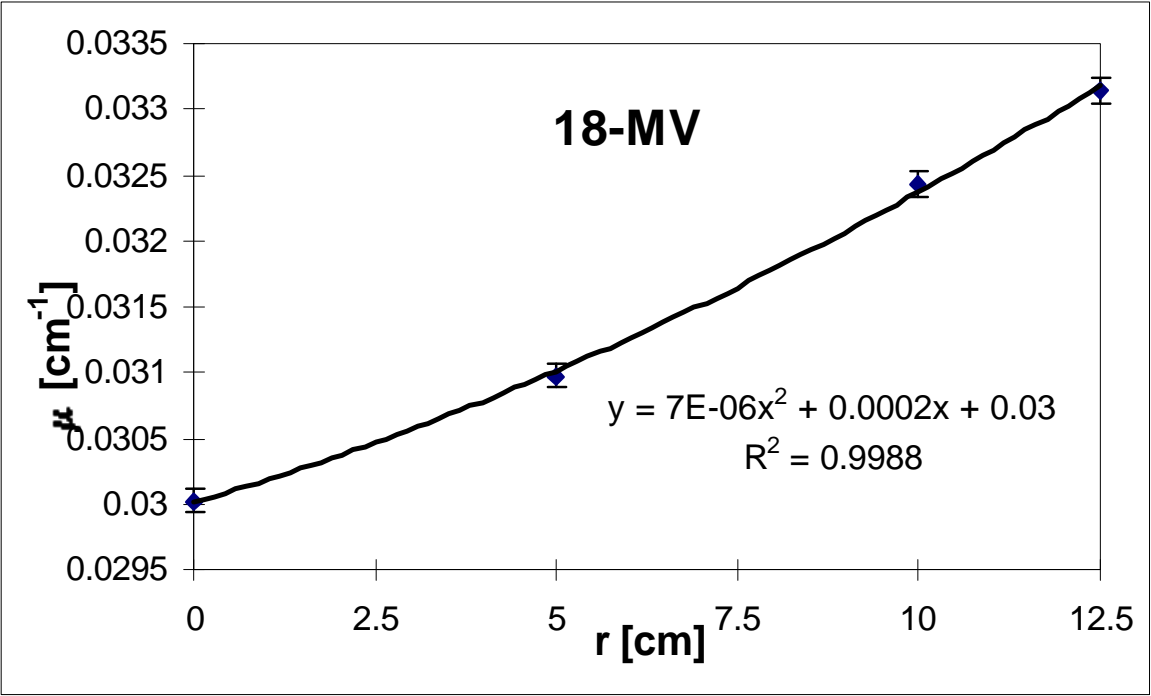


Figure 6.

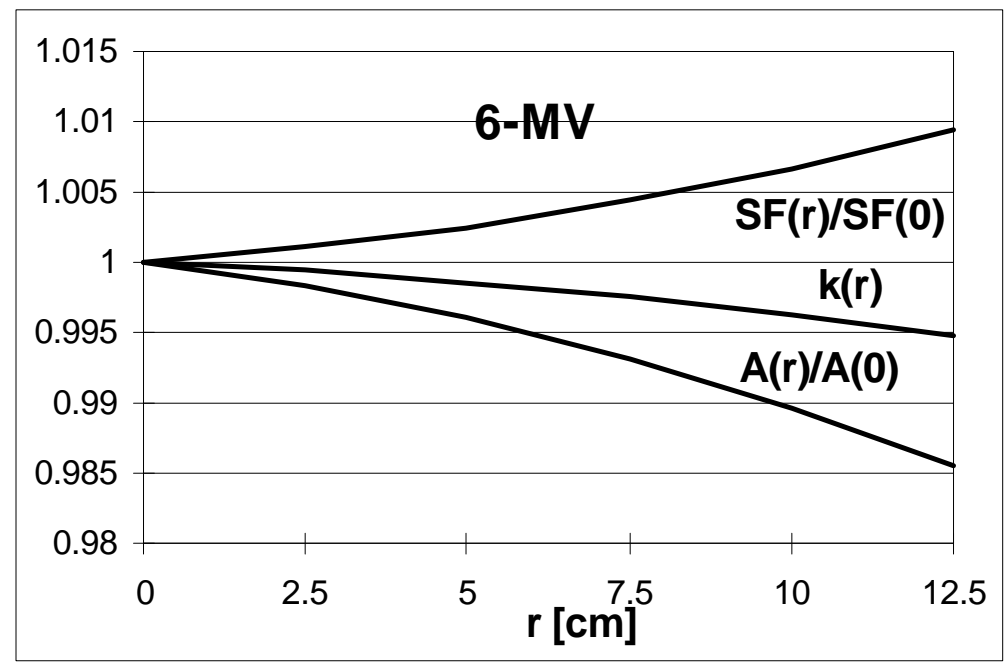

Figure 7.

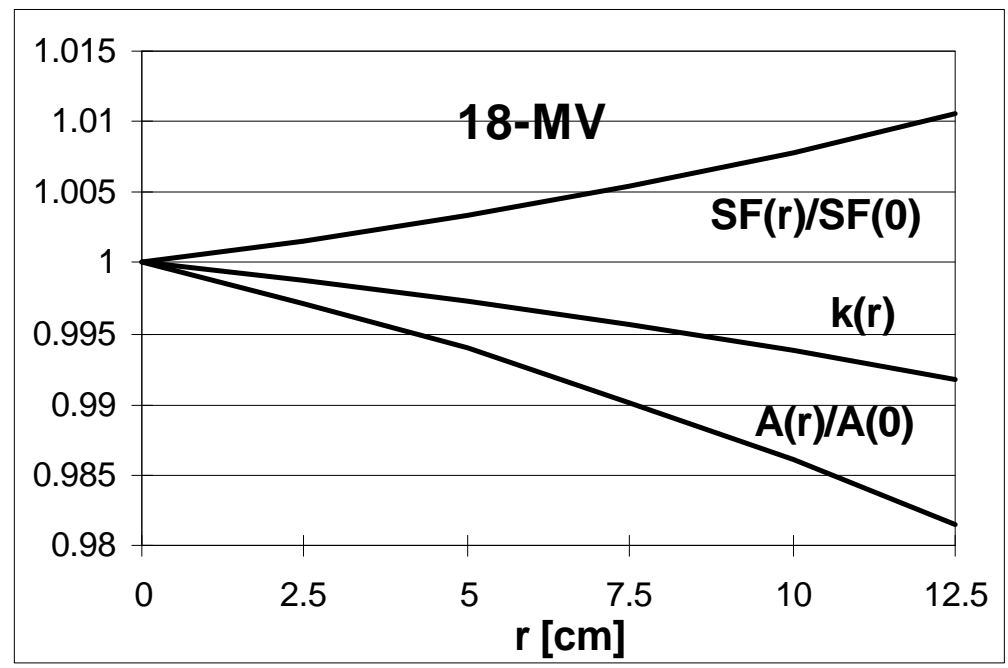

